\title{
A New Approach for Solving Fractional Partial Differential Equations in the Sense of the Modified Riemann-Liouville Derivative
}

\author{
Bin Zheng and Qinghua Feng \\ School of Science, Shandong University of Technology, Zibo, Shandong 255049, China \\ Correspondence should be addressed to Qinghua Feng; fqhua@sina.com
}

Received 26 June 2014; Accepted 2 September 2014; Published 30 September 2014

Academic Editor: Alessandro Palmeri

Copyright (C) 2014 B. Zheng and Q. Feng. This is an open access article distributed under the Creative Commons Attribution License, which permits unrestricted use, distribution, and reproduction in any medium, provided the original work is properly cited.

\begin{abstract}
Based on a fractional complex transformation, certain fractional partial differential equation in the sense of the modified RiemannLiouville derivative is converted into another ordinary differential equation of integer order, and the exact solutions of the latter are assumed to be expressed in a polynomial in Jacobi elliptic functions including the Jacobi sine function, the Jacobi cosine function, and the Jacobi elliptic function of the third kind. The degree of the polynomial can be determined by the homogeneous balance principle. With the aid of mathematical software, a series of exact solutions for the fractional partial differential equation can be found. For demonstrating the validity of this approach, we apply it to solve the space fractional KdV equation and the space-time fractional Fokas equation. As a result, some Jacobi elliptic functions solutions for the two equations are obtained.
\end{abstract}

\section{Introduction}

Fractional differential equations involving fractional derivatives are generalizations of classical differential equations of integer order. Fractional derivative is useful in describing the memory and hereditary properties of materials and processes. Fractional partial differential equations (FPDEs) are widely used as models to express many important physical phenomena such as fluid mechanics, plasma physics, optical fibers, biology, solid state physics, chemical kinematics, and chemical physics. Recently, there have been a lot of achievements on the theory and applications of fractional partial differential equations and, among these investigations for the properties of fractional partial differential equations, the analytical solutions of FPDEs play an important role in nonlinear science and engineering and can help understand some physical phenomena better. So far many powerful methods have been proposed to seek analytical solutions or semianalytical solutions of fractional partial differential equations. For example, these methods include the simplest equation method [1], the Exp-function method [2], the variational iterative method [3-6], the Adomian decomposition method $[7,8]$, the fractional subequation method [9-12], and the homotopy perturbation method [13-15].

In this paper, we present a new method to seek exact solutions in the forms of the Jacobi elliptic functions for fractional partial differential equations. The fractional partial differential equation is defined in the sense of the modified Riemann-Liouville derivative. This method belongs to the categories of the subequation methods. First by a fractional complex transformation, certain fractional partial differential equation is converted into another ordinary differential equation of integer order. The main point of this method lies in the fact that the solutions of the converted ordinary differential equation are supposed to be expressed in a polynomial in Jacobi elliptic functions, and the degree of the polynomial can be determined by the homogeneous balance principle. By substituting this polynomial into the ordinary differential equation, a series of algebraic equations can be derived. By solving these equations with mathematical software such as Maple, a series of exact solutions in the forms of the Jacobi elliptic functions can be obtained.

The modified Riemann-Liouville fractional derivative, defined by Jumarie in [16-19], has many excellent characters 
in handling with many fractional calculus problems. We now list the definition for it as follows.

Definition 1. The modified Riemann-Liouville derivative of order $\alpha$ is defined by the following expression:

$$
D_{t}^{\alpha} f(t)=\left\{\begin{array}{lr}
\frac{1}{\Gamma(1-\alpha)} \frac{d}{d t} \int_{0}^{t}(t-\xi)^{-\alpha}(f(\xi)-f(0)) d \xi, \\
\left(f^{(n)}(t)\right)^{(\alpha-n)}, & 0<\alpha<1, \\
& n \leq \alpha<n+1, n \geq 1 .
\end{array}\right.
$$

Some important properties for the modified RiemannLiouville derivative are listed as follows (see $[8-11,15])$ :

$$
\begin{gathered}
D_{t}^{\alpha} t^{r}=\frac{\Gamma(1+r)}{\Gamma(1+r-\alpha)} t^{r-\alpha}, \\
D_{t}^{\alpha}(f(t) g(t))=g(t) D_{t}^{\alpha} f(t)+f(t) D_{t}^{\alpha} g(t), \\
D_{t}^{\alpha} f[g(t)]=f_{g}^{\prime}[g(t)] D_{t}^{\alpha} g(t), \\
D_{t}^{\alpha} f[g(t)]=D_{g}^{\alpha} f[g(t)]\left(g^{\prime}(t)\right)^{\alpha} .
\end{gathered}
$$

We organize the rest of this paper as follows. In Section 2, we give the description of the present method for seeking Jacobi elliptic function solutions for fractional partial differential equations. Then in Section 3 we present some applications and apply this method to the space fractional KdV equation and the space-time fractional Fokas equation. Some concluding comments are presented in Section 4.

\section{Summary of the Method}

In this section, we give the description of the method for solving seeking Jacobi elliptic function solutions for fractional partial differential equations.

Suppose that a fractional partial differential equation, say in the independent variables $t, x_{1}, x_{2}, \ldots, x_{n}$, is given by

$$
\begin{gathered}
P\left(u_{1}, \ldots, u_{k}, D_{t}^{\alpha} u_{1}, \ldots, D_{t}^{\alpha} u_{k}, \frac{\partial u_{1}}{\partial x_{1}}, \ldots, \frac{\partial u_{k}}{\partial x_{1}},\right. \\
D_{x_{2}}^{\beta} u_{1}, \ldots, D_{x_{2}}^{\beta} u_{1}, \ldots, \frac{\partial u_{1}}{\partial x_{n-1}}, \ldots, \\
\left.\frac{\partial u_{k}}{\partial x_{n-1}}, D_{x_{n}}^{\gamma} u_{1}, \ldots, D_{x_{n}}^{\gamma} u_{k}, \ldots\right)=0
\end{gathered}
$$

where $u_{i}=u_{i}\left(t, x_{1}, x_{2}, \ldots, x_{n}\right), i=1, \ldots, k$, are unknown functions, $P$ is a polynomial in $u_{i}$, and their various partial derivatives include fractional derivatives.
Step 1. For (6), suppose that $u_{i}\left(t, x_{1}, x_{2}, \ldots, x_{n}\right)=U_{i}(\xi)$ and a fractional complex transformation for $\xi$ as follows:

$$
\begin{aligned}
\xi= & \frac{c t^{\alpha}}{\Gamma(1+\alpha)}+k_{1} x_{1}+\frac{k_{2} x_{2}^{\beta}}{\Gamma(1+\beta)}+\cdots k_{n-1} x_{n-1} \\
& +\frac{k_{n} x_{n}^{\gamma}}{\Gamma(1+\gamma)}+\xi_{0},
\end{aligned}
$$

where $c, k_{1}, \ldots, k_{n-1}, k_{n}, \xi_{0}$ are all nonzero constants. Based on the transformation above, for the terms in (6) containing fractional derivative, such as $D_{t}^{\alpha} u_{1}$, by using (2) and (4), one can obtain that

$$
D_{t}^{\alpha} u_{1}=D_{t}^{\alpha} U_{1}(\xi)=U_{1}^{\prime}(\xi) D_{t}^{\alpha} \xi=c U_{1}^{\prime}(\xi) .
$$

For the terms in (6) containing derivative of integer order, such as $\partial u_{1} / \partial x_{1}$, one has

$$
\frac{\partial u_{1}}{\partial x_{1}}=\frac{\partial U_{1}}{\partial \xi} \xi_{x_{1}}^{\prime}=k_{1} U_{1}^{\prime}(\xi)
$$

So by this transformation for $\xi$, (6) can be turned into the following ordinary differential equation of integer order with respect to the variable $\xi$ :

$$
\widetilde{P}\left(U_{1}, \ldots, U_{k}, U_{1}^{\prime}, \ldots, U_{k}^{\prime}, U_{1}^{\prime \prime}, \ldots, U_{k}^{\prime \prime}, \ldots\right)=0 .
$$

Step 2. Suppose that the solution of (10) can be expressed by a polynomial in Jacobi elliptic functions as follows:

$$
\begin{array}{r}
U_{j}(\xi)=a_{j}^{(0)}+\sum_{n+p+q=1}^{m_{j}} a_{j}^{(n, p, q)} \operatorname{sn}^{n}(\xi) \operatorname{cn}^{p}(\xi) \operatorname{dn}^{q}(\xi), \\
j=1,2, \ldots, k,
\end{array}
$$

where $n, p$, and $q$ are nonnegative integers with $1 \leq n+$ $p+q \leq m_{j}, a_{j}^{(0)}, a_{j}^{(n, p, q)}, j=1,2, \ldots, k$, are constants to be determined later, the positive integer $m_{j}$ can be determined by considering the homogeneous balance between the highest order derivatives, and nonlinear terms appearing in $(10), \operatorname{sn}(\xi), \operatorname{cn}(\xi)$, and $\operatorname{dn}(\xi)$, denote the Jacobi elliptic sine function, Jacobi elliptic cosine function, and the Jacobi elliptic function of the third kind, respectively.

For the Jacobi elliptic functions, one has

$$
\begin{gathered}
\operatorname{sn}^{\prime}(\xi)=\operatorname{cn}(\xi) \operatorname{dn}(\xi), \quad \operatorname{cn}^{\prime}(\xi)=-\operatorname{sn}(\xi) \operatorname{dn}(\xi), \\
\operatorname{dn}^{\prime}(\xi)=-m^{2} \operatorname{sn}(\xi) \operatorname{cn}(\xi),
\end{gathered}
$$

where $m$ is the modulus, and

$$
\begin{array}{rlrl}
\operatorname{cs}(\xi) & =\frac{\operatorname{cn}(\xi)}{\operatorname{sn}(\xi)}, & \operatorname{sd}(\xi)=\frac{\operatorname{sn}(\xi)}{\operatorname{dn}(\xi)}, & \operatorname{dc}(\xi)=\frac{\operatorname{dn}(\xi)}{\operatorname{cn}(\xi)}, \\
\operatorname{sc}(\xi)=\frac{1}{\operatorname{cs}(\xi)}, & \operatorname{ds}(\xi)=\frac{1}{\operatorname{sd}(\xi)}, & \operatorname{cd}(\xi)=\frac{1}{\operatorname{dc}(\xi)}, \\
\operatorname{nd}(\xi)=\frac{1}{\operatorname{dn}(\xi)}, & \operatorname{ns}(\xi)=\frac{1}{\operatorname{sn}(\xi)}, & \operatorname{nc}(\xi)=\frac{1}{\operatorname{cn}(\xi)},
\end{array}
$$




$$
\begin{aligned}
& \mathrm{cn}^{2}(\xi)=-\mathrm{sn}^{2}(\xi)+1, \quad \mathrm{dn}^{2}(\xi)=-m^{2} \mathrm{sn}^{2}(\xi)+1, \\
& \mathrm{dn}^{2}(\xi)=m^{2} \mathrm{cn}^{2}(\xi)+1-m^{2}, \quad \mathrm{~ns}^{2}(\xi)=\mathrm{cs}^{2}(\xi)+1, \\
& \mathrm{~ns}^{2}(\xi)= \mathrm{ds}^{2}(\xi)+m^{2}, \quad \mathrm{ds}^{2}(\xi)=\mathrm{cs}^{2}(\xi)+1-m^{2}, \\
& \mathrm{nc}^{2}(\xi)=\mathrm{sc}^{2}(\xi)+1, \quad \mathrm{dc}^{2}(\xi)=\left(1-m^{2}\right) \mathrm{nc}^{2}(\xi)+m^{2}, \\
& \mathrm{dc}^{2}(\xi)=\left(1-m^{2}\right) \mathrm{sc}^{2}(\xi)+1, \\
& \mathrm{~cd}^{2}(\xi)=\frac{m^{2}-1}{m^{2}} \mathrm{nd}^{2}(\xi)+\frac{1}{m^{2}}, \\
& \operatorname{cd}^{2}(\xi)=\left(m^{2}-1\right) \operatorname{sd}^{2}(\xi)+1, \\
& \operatorname{nd}^{2}(\xi)=m^{2} \operatorname{sd}^{2}(\xi)+1 .
\end{aligned}
$$

Step 3. By substituting (11) into (10) and using (12), the lefthand side of (10) is converted into another polynomial in $\operatorname{sn}^{n}(\xi) \mathrm{cn}^{p}(\xi) \mathrm{dn}^{q}(\xi)$. Collecting all coefficients of the same power and equating them to zero yield a set of algebraic equations for $a_{j}^{(0)}, a_{j}^{(n, p, q)}, j=1,2, \ldots, k$.

Step 4. By solving the equations system in Step 3, we can construct a variety of Jacobi elliptic function solutions for (6).

\section{Application of the Present Method to Some Fractional Partial Differential Equations}

3.1. Space Fractional KdV Equation. Consider the following space fractional $\mathrm{KdV}$ equation:

$$
u_{t}+u D_{x}^{\alpha} u+\beta D_{x}^{3 \alpha} u=0, \quad 0<\alpha \leq 1,
$$

where $u=u(x, t)$ is the distribution function in terms of the space $x$ and the time $t$, the concerned fractional derivative is defined by the modified Riemann-Liouville derivative, and $\beta$ is a constant denoting the dispersion coefficient. When $\alpha=1$, (14) becomes the known $\mathrm{KdV}$ equation of integer order, which can be used to describe the motions of waves in nonlinear optics, plasma, or fluids.

In the following, we will apply the described method in Section 2 to solve (14) and seek Jacobi elliptic function solutions for it. To this end, we suppose $u(x, t)=U(\xi)$, where $\xi=c t+(k / \Gamma(1+\alpha)) x^{\alpha}+\xi_{0}, k, c, \xi_{0}$ are all constants with $k$, $c \neq 0$. Then by the use of (2) and (4) one can deduce that

$$
\begin{aligned}
D_{x}^{\alpha} u=D_{x}^{\alpha} U(\xi) & =U^{\prime}(\xi) D_{x}^{\alpha} \xi=k_{1} U^{\prime}(\xi), \\
u_{t} & =c U^{\prime}(\xi),
\end{aligned}
$$

and then (14) can be turned into the following form with respect to the new variable $\xi$ :

$$
U^{\prime}(\xi)+k U(\xi) U^{\prime}(\xi)+\beta k^{3} U^{\prime \prime \prime}(\xi)=0 .
$$

Suppose that the solution of (16) can be expressed by

$$
U(\xi)=a^{(0)}+\sum_{n+p+q=1}^{m} a^{(n, p, q)} \operatorname{sn}^{n}(\xi) \operatorname{cn}^{p}(\xi) \mathrm{dn}^{q}(\xi) .
$$

By balancing the order of $U^{\prime \prime \prime}$ and $U U^{\prime}$ in (16) one can obtain $m=2$. So

$$
\begin{aligned}
U(\xi)= & a^{(0)}+a^{(1,0,0)} \operatorname{sn}(\xi)+a^{(0,1,0)} \operatorname{cn}(\xi)+a^{(0,0,1)} \operatorname{dn}(\xi) \\
& +a^{(2,0,0)} \operatorname{sn}^{2}(\xi)+a^{(0,2,0)} \operatorname{cn}^{2}(\xi)+a^{(0,0,2)} \operatorname{dn}^{2}(\xi) \\
& +a^{(1,1,0)} \operatorname{sn}(\xi) \operatorname{cn}(\xi)+a^{(0,1,1)} \operatorname{cn}(\xi) \operatorname{dn}(\xi) \\
& +a^{(1,0,1)} \operatorname{sn}(\xi) \operatorname{dn}(\xi) .
\end{aligned}
$$

Substituting (18) into (16), using (12) and collecting all the terms with the same power of $\operatorname{sn}^{n}(\xi) \mathrm{cn}^{p}(\xi) \operatorname{dn}^{q}(\xi)$ together, equating each coefficient to zero yields a set of algebraic equations. Solving these equations with the aid of mathematical software yields the following values, where $i$ denoted the unit of the imaginary numbers.

Case 1. Consider

$$
\begin{gathered}
a^{(0)}=-\frac{c}{k}, \quad a^{(1,0,0)}=0, \quad a^{(0,1,0)}=0, \\
a^{(0,0,1)}=0, \quad a^{(2,0,0)}=-4 \beta k^{2} m^{2}, \quad a^{(0,2,0)}=4 \beta k^{2} m^{2}, \\
a^{(0,0,2)}=4 \beta k^{2}, \quad a^{(1,1,0)}=0, \\
a^{(0,1,1)}=0, \quad a^{(1,0,1)}=0 .
\end{gathered}
$$

Case 2. Consider

$$
\begin{aligned}
& a^{(0)}=-\frac{c}{k}, \quad a^{(1,0,0)}=0, \quad a^{(0,1,0)}=0, \quad a^{(0,0,1)}=0, \\
& a^{(2,0,0)}=-4 \beta k^{2} m^{2}, \quad a^{(0,2,0)}=\beta k^{2} m^{2}, \quad a^{(0,0,2)}=\beta k^{2}, \\
& a^{(1,1,0)}=0, \quad a^{(0,1,1)}= \pm 6 \beta k^{2} m, \quad a^{(1,0,1)}=0 .
\end{aligned}
$$

Case 3. Consider

$$
\begin{aligned}
& a^{(0)}=-\frac{c}{k}, \quad a^{(1,0,0)}=0, \quad a^{(0,1,0)}=0, \quad a^{(0,0,1)}=0, \\
& a^{(2,0,0)}=-\beta k^{2} m^{2}, \quad a^{(0,2,0)}=\beta k^{2} m^{2}, \quad a^{(0,0,2)}=4 \beta k^{2}, \\
& a^{(1,1,0)}= \pm 6 m^{2} k^{2} \beta i, \quad a^{(0,1,1)}=0, \quad a^{(1,0,1)}=0 .
\end{aligned}
$$

Case 4. Consider

$$
\begin{aligned}
& a^{(0)}=-\frac{c}{k}, \quad a^{(1,0,0)}=0, \quad a^{(0,1,0)}=0, \quad a^{(0,0,1)}=0, \\
& a^{(2,0,0)}=-\beta k^{2} m^{2}, \quad a^{(0,2,0)}=4 \beta k^{2} m^{2}, \quad a^{(0,0,2)}=\beta k^{2}, \\
& a^{(1,1,0)}= \pm 6 m k^{2} \beta i, \quad a^{(0,1,1)}=0, \quad a^{(1,0,1)}=0 .
\end{aligned}
$$


Case 5. Consider

$$
\begin{gathered}
a^{(0)}=-\frac{c}{k}, \quad a^{(1,0,0)}=0, \quad a^{(0,1,0)}=0, \quad a^{(0,0,1)}=0, \\
a^{(2,0,0)}=-\beta k^{2} m^{2}, \quad a^{(0,2,0)}=\beta k^{2} m^{2}, \quad a^{(0,0,2)}=\beta k^{2}, \\
a^{(1,1,0)}= \pm 3 m^{2} k^{2} \beta i, \quad a^{(0,1,1)}= \pm 3 \beta k^{2} m, \\
a^{(1,0,1)}= \pm 3 \beta k^{2} m i .
\end{gathered}
$$

Case 6. Consider

$$
\begin{gathered}
a^{(0)}=a^{(0)}, \quad a^{(1,0,0)}=0, \quad a^{(0,1,0)}=0, \quad a^{(0,0,1)}=0, \\
a^{(2,0,0)}=a^{(0,2,0)}+m^{2} a^{(0,0,2)}, \quad a^{(0,2,0)}=a^{(0,2,0)}, \\
a^{(0,0,2)}=a^{(0,0,2)}, \quad a^{(1,1,0)}=0, \quad a^{(0,1,1)}=0, \\
a^{(1,0,1)}=0,
\end{gathered}
$$

where $a^{(0)}, a^{(0,2,0)}$, and $a^{(0,0,2)}$ are arbitrary constants.

By substituting the results above into (18) we can obtain the following exact solutions in the forms of the Jacobi elliptic functions for (14), where $\xi=c t+(k / \Gamma(1+\alpha)) x^{\alpha}+\xi_{0}$.

Family 1. Consider

$$
\begin{aligned}
u_{1}(x, t)= & -\frac{c}{k}-4 \beta k^{2} m^{2} \operatorname{sn}^{2}(\xi)+4 \beta k^{2} m^{2} \mathrm{cn}^{2}(\xi) \\
& +4 \beta k^{2} \operatorname{dn}^{2}(\xi)
\end{aligned}
$$

Family 2. Consider

$$
\begin{aligned}
u_{2}(x, t)= & -\frac{c}{k}-4 \beta k^{2} m^{2} \operatorname{sn}^{2}(\xi)+\beta k^{2} m^{2} \mathrm{cn}^{2}(\xi) \\
& +\beta k^{2} \mathrm{dn}^{2}(\xi) \pm 6 \beta k^{2} m \mathrm{cn}(\xi) \mathrm{dn}(\xi) .
\end{aligned}
$$

Family 3. Consider

$$
\begin{aligned}
u_{3}(x, t)= & -\frac{c}{k}-\beta k^{2} m^{2} \mathrm{sn}^{2}(\xi)+\beta k^{2} m^{2} \mathrm{cn}^{2}(\xi) \\
& +4 \beta k^{2} \mathrm{dn}^{2}(\xi) \pm 6 m^{2} k^{2} \beta i \operatorname{sn}(\xi) \mathrm{cn}(\xi)
\end{aligned}
$$

Family 4. Consider

$$
\begin{aligned}
u_{4}(x, t)= & -\frac{c}{k}-\beta k^{2} m^{2} \operatorname{sn}^{2}(\xi)+4 \beta k^{2} m^{2} \mathrm{cn}^{2}(\xi) \\
& +\beta k^{2} \mathrm{dn}^{2}(\xi) \pm 6 m k^{2} \beta i \operatorname{sn}(\xi) \mathrm{cn}(\xi) .
\end{aligned}
$$

Family 5. Consider

$$
\begin{aligned}
u_{5}(x, t)= & -\frac{c}{k}-\beta k^{2} m^{2} \operatorname{sn}^{2}(\xi)+\beta k^{2} m^{2} \mathrm{cn}^{2}(\xi) \\
& +\beta k^{2} \operatorname{dn}^{2}(\xi) \pm 3 m^{2} k^{2} \beta i \operatorname{sn}(\xi) \mathrm{cn}(\xi) \\
& \pm 3 \beta k^{2} m \mathrm{cn}(\xi) \mathrm{dn}(\xi) \pm 3 \beta k^{2} m i \operatorname{sn}(\xi) \mathrm{dn}(\xi)
\end{aligned}
$$

Family 6. Consider

$$
\begin{aligned}
u_{6}(x, t)= & a^{(0)}+\left[a^{(0,2,0)}+m^{2} a^{(0,0,2)}\right] \mathrm{sn}^{2}(\xi)+a^{(0,2,0)} \mathrm{cn}^{2}(\xi) \\
& +a^{(0,0,2)} \mathrm{dn}^{2}(\xi)
\end{aligned}
$$

where $a^{(0)}, a^{(0,2,0)}$, and $a^{(0,0,2)}$ are arbitrary constants.

Remark 2. We note that the Jacobi elliptic function solutions established in (25)-(30) for the space fractional KdV equation (14) are new exact solutions so far in the literature.

3.2. Space-Time Fractional Fokas Equation. Consider the following space-time fractional Fokas equation $[8,20,21]$ :

$$
\begin{aligned}
& 4 \frac{\partial^{2 \alpha} q}{\partial t^{\alpha} \partial x_{1}^{\alpha}}-\frac{\partial^{4 \alpha} q}{\partial x_{1}^{3 \alpha} \partial x_{2}^{\alpha}}+\frac{\partial^{4 \alpha} q}{\partial x_{2}^{3 \alpha} \partial x_{1}^{\alpha}}+12 \frac{\partial^{\alpha} q}{\partial x_{1}^{\alpha}} \frac{\partial^{\alpha} q}{\partial x_{2}^{\alpha}} \\
& +12 q \frac{\partial^{2 \alpha} q}{\partial x_{1}^{\alpha} \partial x_{2}^{\alpha}}-6 \frac{\partial^{2 \alpha} q}{\partial y_{1}^{\alpha} \partial y_{2}^{\alpha}}=0, \quad 0<\alpha \leq 1 .
\end{aligned}
$$

Equation (31) is a transformed generalization of the following $(4+1)$-dimensional Fokas equation:

$$
\frac{\partial^{2} q}{\partial t \partial x_{1}}=\frac{1}{4} \frac{\partial^{4} q}{\partial x_{1}^{3} \partial x_{2}}-\frac{1}{4} \frac{\partial^{4} q}{\partial x_{2}^{3} \partial x_{1}}-\frac{3}{2} q \frac{\partial^{2} q^{2}}{\partial x_{1} \partial x_{2}}+\frac{3}{2} \frac{\partial^{2} q}{\partial y_{1} \partial y_{2}}
$$

which is one of the new high-dimensional nonlinear wave equations Fokas recently obtained by extending the integrable KP equation and DS equation. In [8], El-Sayed et al. obtained some exact analytical solutionsfor (31) including the generalized hyperbolic function solutions, generalized trigonometric function solutions, and rational solutions, while in $[20,21]$ the authors obtained some other new exact solutions using the Riccati equation method and the $\left(D^{\alpha} G / G\right)$ method.

In the following we will apply the proposed method in Section 2 to solve (31).

Suppose $q\left(t, x_{1}, x_{2}, y_{1}, y_{2}\right)=Q(\xi)$, where $\xi=k_{1} x_{1}^{\alpha} / \Gamma(1+$ $\alpha)+k_{2} x_{2}^{\alpha} / \Gamma(1+\alpha)+l_{1} y_{1}^{\alpha} / \Gamma(1+\alpha)+l_{2} y_{2}^{\alpha} / \Gamma(1+\alpha)+c t^{\alpha} / \Gamma(1+\alpha)+$ $\xi_{0} ; k_{1}, k_{2}, l_{1}, l_{2}, c, \xi_{0}$ are all constants with $k_{1}, k_{2}, l_{1}, l_{2}, c \neq 0$. Then by the use of (2) and (4), and similar to the process of (15) and (16), (31) can be converted into the following form:

$$
\begin{gathered}
4 c k_{1} U^{\prime \prime}-k_{1}^{3} k_{2} U^{(4)}+k_{2}^{3} k_{1} U^{(4)}+12 k_{1} k_{2} U^{\prime 2} \\
+12 k_{1} k_{2} U U^{\prime \prime}-6 l_{1} l_{2} U^{\prime \prime}=0 .
\end{gathered}
$$

Suppose that the solution of (33) can be expressed by

$$
Q(\xi)=a^{(0)}+\sum_{n+p+q=1}^{m} a^{(n, p, q)} \operatorname{sn}^{n}(\xi) \mathrm{cn}^{p}(\xi) \mathrm{dn}^{q}(\xi)
$$


By balancing the order of $U^{(4)}$ and $U U^{\prime \prime}$ in (33) one can obtain $m=2$. So

$$
\begin{aligned}
Q(\xi)= & a^{(0)}+a^{(1,0,0)} \operatorname{sn}(\xi)+a^{(0,1,0)} \operatorname{cn}(\xi) \\
& +a^{(0,0,1)} \operatorname{dn}(\xi)+a^{(2,0,0)} \operatorname{sn}^{2}(\xi)+a^{(0,2,0)} \mathrm{cn}^{2}(\xi) \\
& +a^{(0,0,2)} \operatorname{dn}^{2}(\xi)+a^{(1,1,0)} \operatorname{sn}(\xi) \operatorname{cn}(\xi) \\
& +a^{(0,1,1)} \operatorname{cn}(\xi) \operatorname{dn}(\xi)+a^{(1,0,1)} \operatorname{sn}(\xi) \operatorname{dn}(\xi) .
\end{aligned}
$$

Substituting (35) into (33), using (12) and collecting all the terms with the same power of $\operatorname{sn}^{n}(\xi) \mathrm{cn}^{p}(\xi) \mathrm{dn}^{q}(\xi)$ together, equating each coefficient to zero yields a set of algebraic equations. Solving these equations yields the following values.

Case 1. Consider

$$
\begin{gathered}
a^{(0)}=-\frac{2 c k_{1}-3 l_{1} l_{2}}{6 k_{1} k_{2}}, \quad a^{(1,0,0)}=0, \quad a^{(0,1,0)}=0, \\
a^{(0,0,1)}=0, \quad a^{(2,0,0)}=\frac{1}{3} m^{2}\left(k_{1}^{2}-k_{2}^{2}\right), \\
a^{(0,2,0)}=\frac{1}{3} m^{2}\left(k_{2}^{2}-k_{1}^{2}\right), \quad a^{(0,0,2)}=\frac{1}{3}\left(k_{2}^{2}-k_{1}^{2}\right), \\
a^{(1,1,0)}=0, \quad a^{(0,1,1)}=0, \quad a^{(1,0,1)}=0 .
\end{gathered}
$$

Case 2. Consider

$$
\begin{gathered}
a^{(0)}=-\frac{2 c k_{1}-3 l_{1} l_{2}}{6 k_{1} k_{2}}, \quad a^{(1,0,0)}=0, \quad a^{(0,1,0)}=0, \\
a^{(0,0,1)}=0, \quad a^{(2,0,0)}=\frac{1}{3} m^{2}\left(k_{1}^{2}-k_{2}^{2}\right), \\
a^{(0,2,0)}=\frac{1}{12} m^{2}\left(k_{2}^{2}-k_{1}^{2}\right), \quad a^{(0,0,2)}=\frac{1}{12}\left(k_{2}^{2}-k_{1}^{2}\right), \\
a^{(1,1,0)}=0, \quad a^{(0,1,1)}= \pm \frac{1}{2} m\left(k_{1}^{2}-k_{2}^{2}\right), \quad a^{(1,0,1)}=0 .
\end{gathered}
$$

Case 3. Consider

$$
\begin{gathered}
a^{(0)}=-\frac{2 c k_{1}-3 l_{1} l_{2}}{6 k_{1} k_{2}}, \quad a^{(1,0,0)}=0, \quad a^{(0,1,0)}=0, \\
a^{(0,0,1)}=0, \quad a^{(2,0,0)}=\frac{1}{12} m^{2}\left(k_{1}^{2}-k_{2}^{2}\right), \\
a^{(0,2,0)}=\frac{1}{12} m^{2}\left(k_{2}^{2}-k_{1}^{2}\right), \quad a^{(0,0,2)}=\frac{1}{3}\left(k_{2}^{2}-k_{1}^{2}\right), \\
a^{(1,1,0)}= \pm \frac{i}{2}\left(k_{1}^{2}-k_{2}^{2}\right) m^{2}, \quad a^{(0,1,1)}=0, \quad a^{(1,0,1)}=0 .
\end{gathered}
$$

Case 4. Consider

$$
\begin{gathered}
a^{(0)}=-\frac{2 c k_{1}-3 l_{1} l_{2}}{6 k_{1} k_{2}}, \quad a^{(1,0,0)}=0, \quad a^{(0,1,0)}=0, \\
a^{(0,0,1)}=0, \quad a^{(2,0,0)}=\frac{1}{12} m^{2}\left(k_{1}^{2}-k_{2}^{2}\right), \\
a^{(0,2,0)}=\frac{1}{3} m^{2}\left(k_{2}^{2}-k_{1}^{2}\right), \quad a^{(0,0,2)}=\frac{1}{12}\left(k_{2}^{2}-k_{1}^{2}\right), \\
a^{(1,1,0)}=0, \quad a^{(0,1,1)}=0, \quad a^{(1,0,1)}= \pm \frac{i}{2} m\left(k_{1}^{2}-k_{2}^{2}\right) .
\end{gathered}
$$

Case 5. Consider

$$
\begin{gathered}
a^{(0)}=-\frac{2 c k_{1}-3 l_{1} l_{2}}{6 k_{1} k_{2}}, \quad a^{(1,0,0)}=0, \quad a^{(0,1,0)}=0, \\
a^{(0,0,1)}=0, \quad a^{(2,0,0)}=\frac{1}{12} m^{2}\left(k_{1}^{2}-k_{2}^{2}\right), \\
a^{(0,2,0)}=\frac{1}{12} m^{2}\left(k_{2}^{2}-k_{1}^{2}\right), \quad a^{(0,0,2)}=\frac{1}{12}\left(k_{2}^{2}-k_{1}^{2}\right), \\
a^{(1,1,0)}= \pm \frac{i}{4}\left(k_{1}^{2}-k_{2}^{2}\right) m^{2}, \quad a^{(0,1,1)}=\mp \frac{1}{4} m\left(k_{1}^{2}-k_{2}^{2}\right), \\
a^{(1,0,1)}= \pm \frac{i}{4} m\left(k_{1}^{2}-k_{2}^{2}\right) .
\end{gathered}
$$

By substituting the results above into (35) we can obtain the following exact solutions in the forms of the Jacobi elliptic functions for (31), where $\xi=k_{1} x_{1}^{\alpha} / \Gamma(1+\alpha)+$ $k_{2} x_{2}^{\alpha} / \Gamma(1+\alpha)+l_{1} y_{1}^{\alpha} / \Gamma(1+\alpha)+l_{2} y_{2}^{\alpha} / \Gamma(1+\alpha)+c t^{\alpha} / \Gamma(1+\alpha)+\xi_{0}$.

Family 1. Consider

$$
\begin{aligned}
q_{1}\left(t, x_{1}, x_{2}, y_{1}, y_{2}\right) \\
=-\frac{2 c k_{1}-3 l_{1} l_{2}}{6 k_{1} k_{2}}+\frac{1}{3} m^{2}\left(k_{1}^{2}-k_{2}^{2}\right) \mathrm{sn}^{2}(\xi) \\
\quad+\frac{1}{3} m^{2}\left(k_{2}^{2}-k_{1}^{2}\right) \mathrm{cn}^{2}(\xi)+\frac{1}{3}\left(k_{2}^{2}-k_{1}^{2}\right) \mathrm{dn}^{2}(\xi) .
\end{aligned}
$$

Family 2. Consider

$$
\begin{aligned}
& q_{2}\left(t, x_{1}, x_{2}, y_{1}, y_{2}\right) \\
&=-\frac{2 c k_{1}-3 l_{1} l_{2}}{6 k_{1} k_{2}}+\frac{1}{3} m^{2}\left(k_{1}^{2}-k_{2}^{2}\right) \operatorname{sn}^{2}(\xi) \\
&+\frac{1}{12} m^{2}\left(k_{2}^{2}-k_{1}^{2}\right) \mathrm{cn}^{2}(\xi)+\frac{1}{12}\left(k_{2}^{2}-k_{1}^{2}\right) \mathrm{dn}^{2}(\xi) \\
& \pm \frac{1}{2} m\left(k_{1}^{2}-k_{2}^{2}\right) \mathrm{cn}(\xi) \mathrm{dn}(\xi) .
\end{aligned}
$$


Family 3. Consider

$$
\begin{aligned}
q_{3}\left(t, x_{1}, x_{2}, y_{1}, y_{2}\right) \\
=-\frac{2 c k_{1}-3 l_{1} l_{2}}{6 k_{1} k_{2}}+\frac{1}{12} m^{2}\left(k_{1}^{2}-k_{2}^{2}\right) \mathrm{sn}^{2}(\xi) \\
\quad+\frac{1}{12} m^{2}\left(k_{2}^{2}-k_{1}^{2}\right) \mathrm{cn}^{2}(\xi)+\frac{1}{3}\left(k_{2}^{2}-k_{1}^{2}\right) \mathrm{dn}^{2}(\xi) \\
\quad \pm \frac{i}{2}\left(k_{1}^{2}-k_{2}^{2}\right) m^{2} \operatorname{sn}(\xi) \mathrm{cn}(\xi) .
\end{aligned}
$$

Family 4. Consider

$$
\begin{aligned}
q_{4}\left(t, x_{1}, x_{2}, y_{1}, y_{2}\right) \\
=-\frac{2 c k_{1}-3 l_{1} l_{2}}{6 k_{1} k_{2}}+\frac{1}{12} m^{2}\left(k_{1}^{2}-k_{2}^{2}\right) \mathrm{sn}^{2}(\xi) \\
\quad+\frac{1}{3} m^{2}\left(k_{2}^{2}-k_{1}^{2}\right) \mathrm{cn}^{2}(\xi)+\frac{1}{12}\left(k_{2}^{2}-k_{1}^{2}\right) \mathrm{dn}^{2}(\xi) \\
\quad \pm \frac{i}{2} m\left(k_{1}^{2}-k_{2}^{2}\right) \operatorname{sn}(\xi) \mathrm{dn}(\xi) .
\end{aligned}
$$

Family 5. Consider

$$
\begin{aligned}
q_{5}\left(t, x_{1}, x_{2}, y_{1}, y_{2}\right) \\
=-\frac{2 c k_{1}-3 l_{1} l_{2}}{6 k_{1} k_{2}}+\frac{1}{12} m^{2}\left(k_{1}^{2}-k_{2}^{2}\right) \mathrm{sn}^{2}(\xi) \\
+\frac{1}{12} m^{2}\left(k_{2}^{2}-k_{1}^{2}\right) \mathrm{cn}^{2}(\xi)+\frac{1}{12}\left(k_{2}^{2}-k_{1}^{2}\right) \mathrm{dn}^{2}(\xi) \\
\quad \pm \frac{i}{4}\left(k_{1}^{2}-k_{2}^{2}\right) m^{2} \operatorname{sn}(\xi) \mathrm{cn}(\xi) \\
\quad \mp \frac{1}{4} m\left(k_{1}^{2}-k_{2}^{2}\right) \mathrm{cn}(\xi) \mathrm{dn}(\xi) \\
\quad \pm \frac{i}{4} m\left(k_{1}^{2}-k_{2}^{2}\right) \operatorname{sn}(\xi) \mathrm{dn}(\xi) .
\end{aligned}
$$

Remark 3. The Jacobi elliptic function solutions established in (41)-(45) are new exact solutions to the space-time fractional Fokas equation (31).

\section{Conclusions}

In this paper, we have proposed a new approach for seeking Jacobi elliptic function solutions to fractional partial differential equations. Based on a fractional complex transformation, certain fractional partial differential equation is converted into another ordinary differential equation of integer order, which permits the exact solutions with the forms of Jacobi elliptic functions, and such solutions can be obtained by use of the concept of the subequation method. So in this way, this method belongs to the categories of subequation methods. As for applications, we apply it to seek Jacobi elliptic function solutions for the space fractional $\mathrm{KdV}$ equation and the space-time fractional Fokas equation. With the aid of mathematical software, a series of exact solutions in the forms of Jacobi elliptic functions for them are successfully found.

\section{Conflict of Interests}

The authors declare that there is no conflict of interests regarding the publication of this paper.

\section{Acknowledgments}

This work was partially supported by the Natural Science Foundation of Shandong Province, China (Grant nos. ZR2013AQ009 and 2014ZRB01058), National Training Programs of Innovation and Entrepreneurship for Undergraduates (Grant no. 201310433031), and Doctoral initializing Foundation of Shandong University of Technology, China (Grant no. 4041-413030). The authors would like to thank the anonymous reviewers very much for their valuable suggestions on improving this paper.

\section{References}

[1] N. Taghizadeh, M. Mirzazadeh, M. Rahimian, and M. Akbari, "Application of the simplest equation method to some timefractional partial differential equations," Ain Shams Engineering Journal, vol. 4, no. 4, pp. 897-902, 2013.

[2] B. Zheng, "Exp-function method for solving fractional partial differential equations," The Scientific World Journal, vol. 2013, Article ID 465723, 8 pages, 2013.

[3] J. He, "A new approach to nonlinear partial differential equations," Communications in Nonlinear Science and Numerical Simulation, vol. 2, no. 4, pp. 230-235, 1997.

[4] G. C. Wu, "A fractional variational iteration method for solving fractional nonlinear differential equations," Computers \& Mathematics with Applications, vol. 61, no. 8, pp. 2186-2190, 2011.

[5] S. Guo and L. Mei, "The fractional variational iteration method using He's polynomials," Physics Letters A, vol. 375, no. 3, pp. 309-313, 2011.

[6] G. Wu and E. W. M. Lee, "Fractional variational iteration method and its application," Physics Letters A, vol. 374, no. 25, pp. 2506-2509, 2010.

[7] A. M. A. El-Sayed and M. Gaber, "The Adomian decomposition method for solving partial differential equations of fractal order in finite domains," Physics Letters A, vol. 359, no. 3, pp. 175-182, 2006.

[8] A. M. A. El-Sayed, S. H. Behiry, and W. E. Raslan, "Adomian's decomposition method for solving an intermediate fractional advection-dispersion equation," Computers \& Mathematics with Applications, vol. 59, no. 5, pp. 1759-1765, 2010.

[9] S. Zhang and H. Q. Zhang, "Fractional sub-equation method and its applications to nonlinear fractional PDEs," Physics Letters A, vol. 375, no. 7, pp. 1069-1073, 2011.

[10] S. Guo, L. Mei, Y. Li, and Y. Sun, "The improved fractional sub-equation method and its applications to the space-time fractional differential equations in fluid mechanics," Physics Letters A, vol. 376, no. 4, pp. 407-411, 2012.

[11] B. Lu, "Bäcklund transformation of fractional Riccati equation and its applications to nonlinear fractional partial differential 
equations," Physics Letters A, vol. 376, no. 28-29, pp. 2045-2048, 2012.

[12] B. Tang, Y. N. He, L. L. Wei, and X. Zhang, "A generalized fractional sub-equation method for fractional differential equations with variable coefficients," Physics Letters A, vol. 376, no. 38-39, pp. 2588-2590, 2012.

[13] S. Guo, L. Mei, and Y. Li, "Fractional variational homotopy perturbation iteration method and its application to a fractional diffusion equation," Applied Mathematics and Computation, vol. 219, no. 11, pp. 5909-5917, 2013.

[14] J. H. He, "Homotopy perturbation technique," Computer Methods in Applied Mechanics and Engineering, vol. 178, no. 3-4, pp. 257-262, 1999.

[15] J.-H. He, "A coupling method of a homotopy technique and a perturbation technique for non-linear problems," International Journal of Non-Linear Mechanics, vol. 35, no. 1, pp. 37-43, 2000.

[16] G. Jumarie, "Modified Riemann-Liouville derivative and fractional Taylor series of nondifferentiable functions further results," Computers \& Mathematics with Applications, vol. 51, no. 9-10, pp. 1367-1376, 2006.

[17] G. Jumarie, "Table of some basic fractional calculus formulae derived from a modified Riemann-Liouville derivative for nondifferentiable functions," Applied Mathematics Letters, vol. 22, no. 3, pp. 378-385, 2009.

[18] G. Jumarie, "Cauchy's integral formula via the modified Riemann-Liouville derivative for analytic functions of fractional order," Applied Mathematics Letters, vol. 23, no. 12, pp. 1444-1450, 2010.

[19] G. Jumarie, "Fractional partial differential equations and modified Riemann-Liouville derivative new methods for solution," Journal of Applied Mathematics \& Computing, vol. 24, no. 1-2, pp. 31-48, 2007.

[20] F. Meng, "A new approach for solving fractional partial differential equations," Journal of Applied Mathematics, vol. 2013, Article ID 256823, 5 pages, 2013.

[21] B. Zheng and C. Wen, "Exact solutions for fractional partial differential equations by a new fractional sub-equation method," Advances in Difference Equations, vol. 199, pp. 1-12, 2013. 


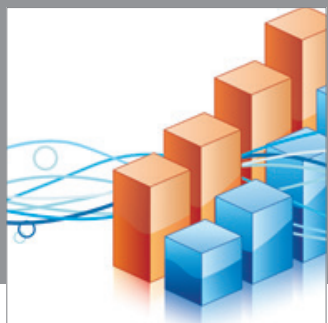

Advances in

Operations Research

mansans

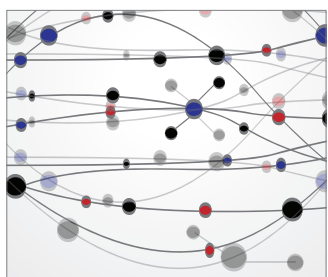

The Scientific World Journal
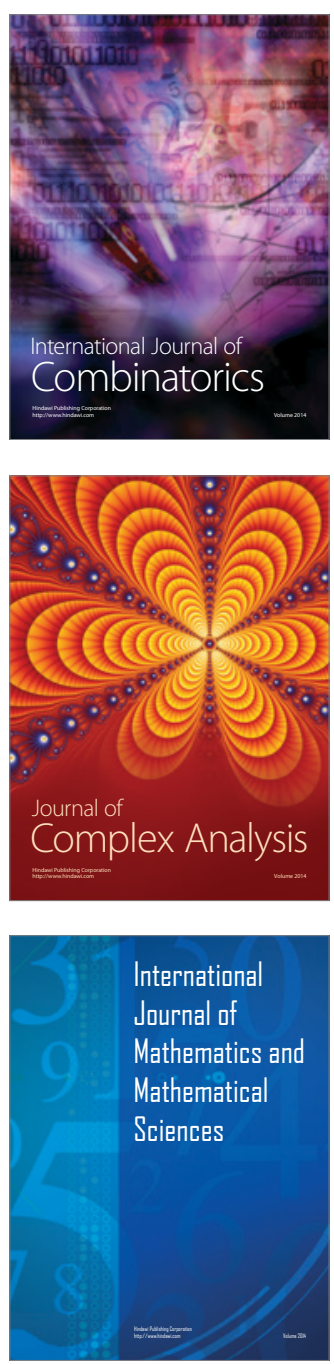
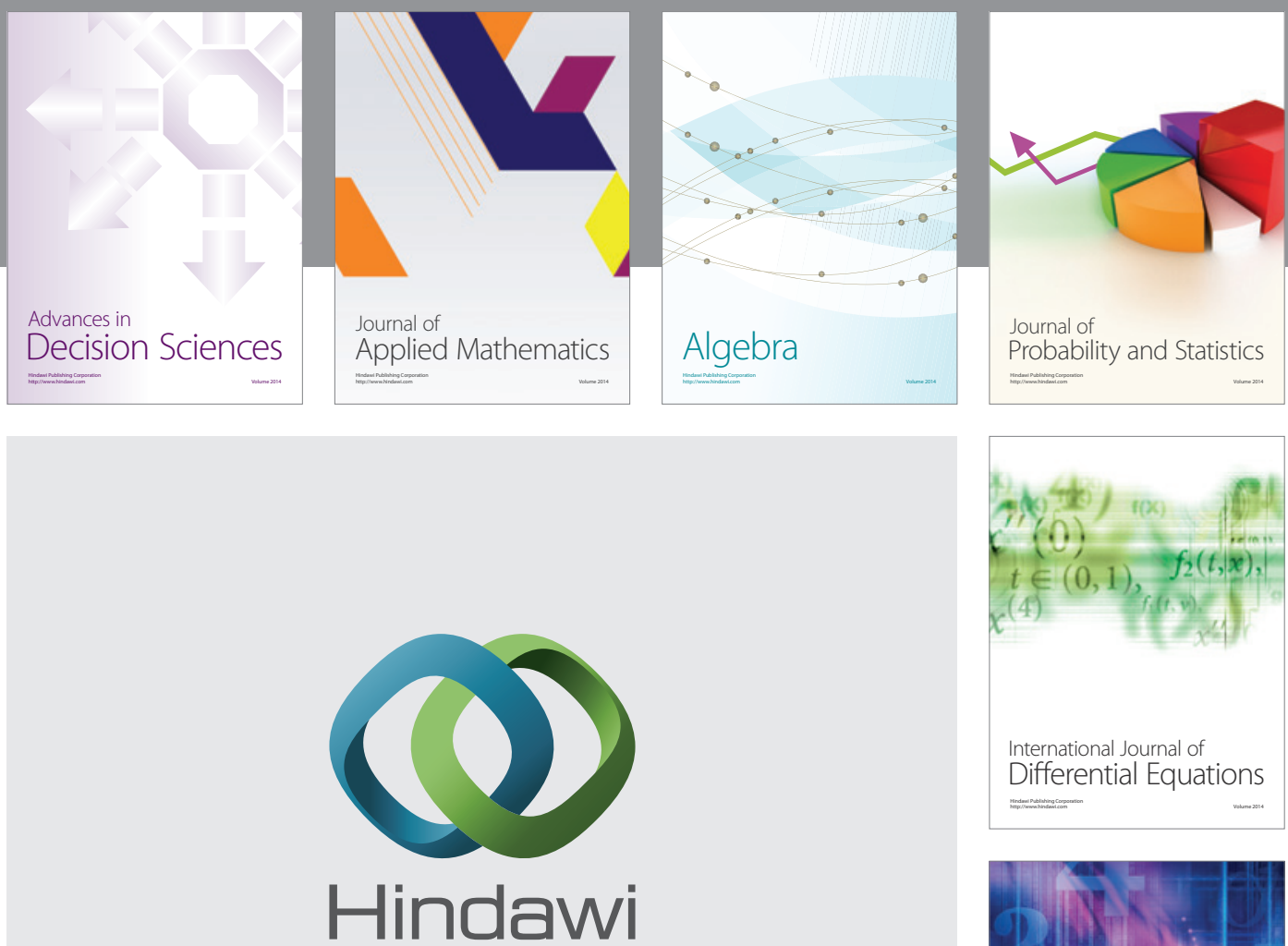

Submit your manuscripts at http://www.hindawi.com
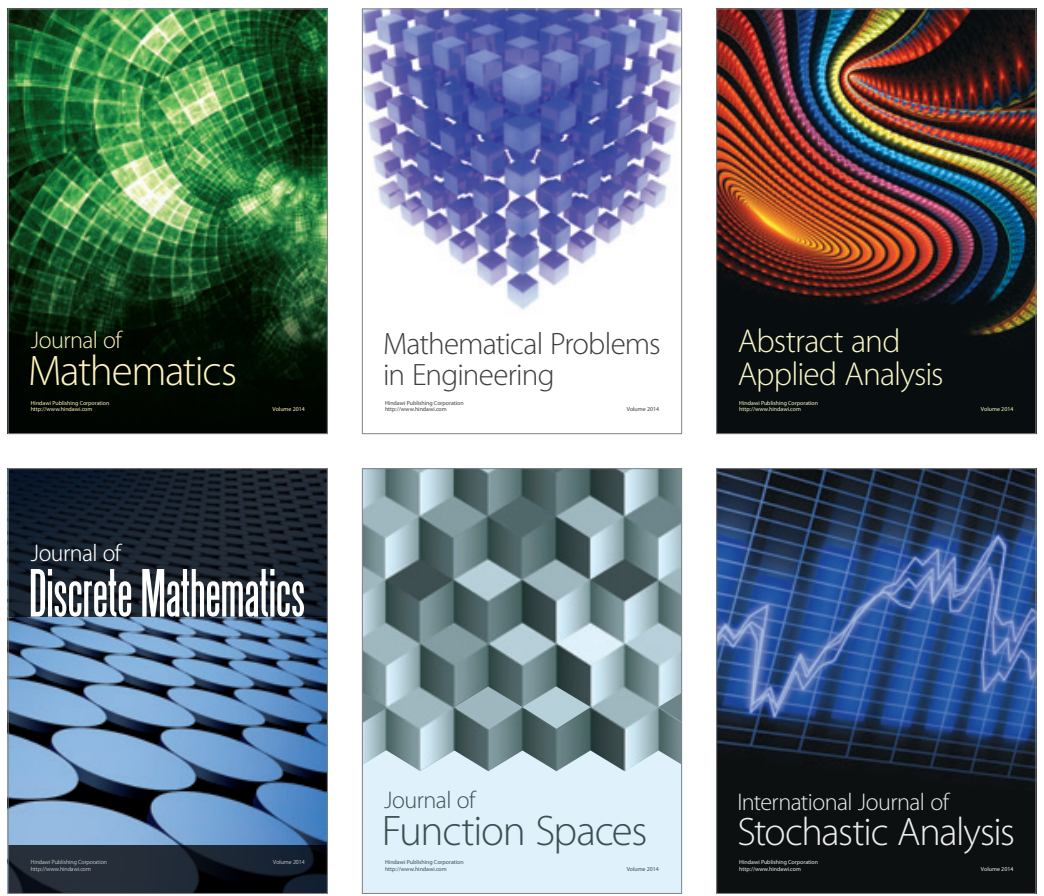

Journal of

Function Spaces

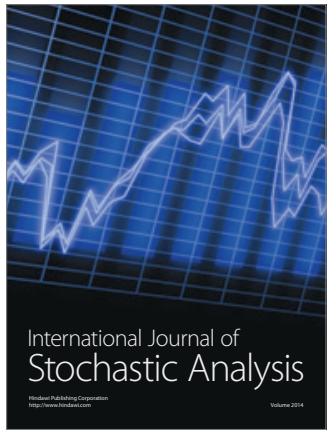

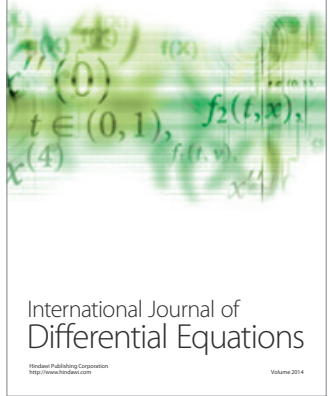
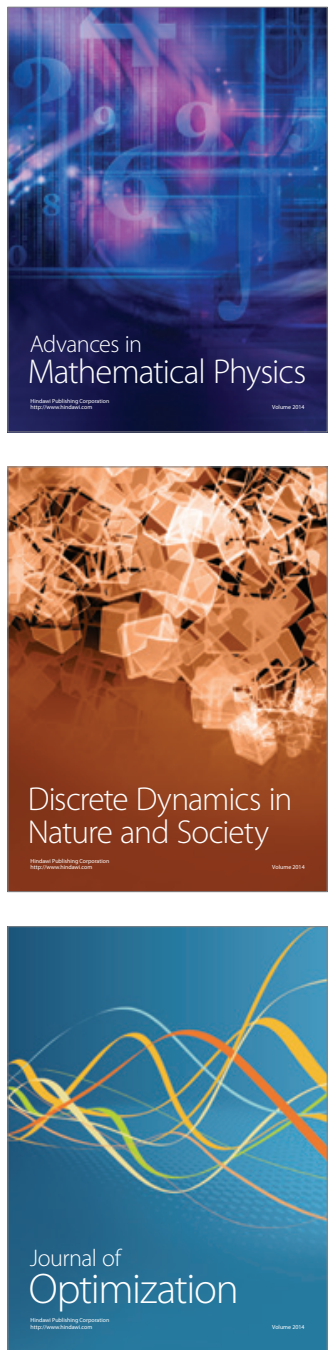